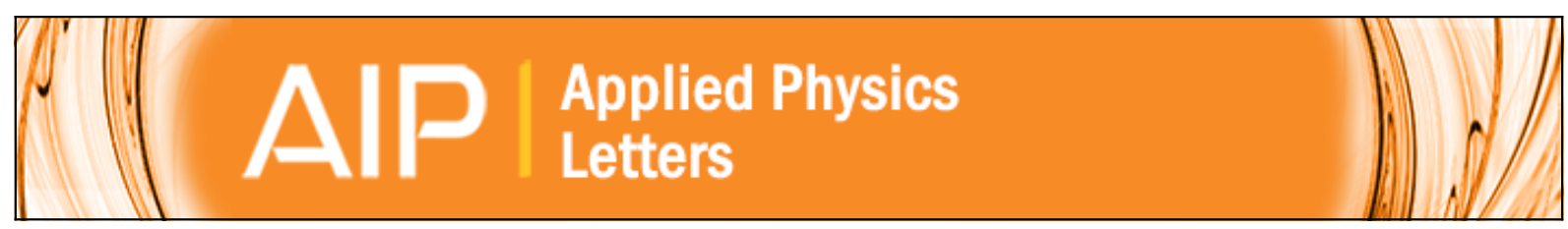

\title{
Dynamic size tuning of multidimensional optically bound matter
}

O. Brzobohatý, V. Karásek, T. ižmár, and P. Zemánek

Citation: Applied Physics Letters 99, 101105 (2011); doi: 10.1063/1.3634007

View online: http://dx.doi.org/10.1063/1.3634007

View Table of Contents: http://scitation.aip.org/content/aip/journal/apl/99/10?ver=pdfcov

Published by the AIP Publishing

$\stackrel{A}{A} \mathbb{P} P$ Re-register for Table of Content Alerts

Create a profile.

Sign up today! 


\title{
Dynamic size tuning of multidimensional optically bound matter
}

\author{
O. Brzobohatý, ${ }^{1}$ V. Karásek, ${ }^{1}$ T. Čižmár, ${ }^{2}$ and P. Zemánek ${ }^{1, a)}$ \\ ${ }^{1}$ Institute of Scientific Instruments of the ASCR, Královopolská 147, Brno 61264, Czech Republic \\ ${ }^{2}$ School of Medicine, University of St. Andrews, North Haugh, Fife KY16 9TF, Scotland
}

(Received 9 May 2011; accepted 8 August 2011; published online 8 September 2011)

\begin{abstract}
We generate and dynamically control one-, two- and three-dimensional optically bound structures of soft matter in the geometry of counter-propagating incoherent laser beams. We report results for the Bessel, Gaussian, and Laguerre-Gaussian laser modes and particularly focus on the influence of the lateral dimensions of the beam profile on the resulting self-arranged optically bound structures. Employing the transfer of the orbital angular momentum of light in the Laguerre-Gaussian beams, we show that optically bound structures can conserve their spatial arrangements even while orbiting along the beam circumference. (C) 2011 American Institute of Physics. [doi:10.1063/1.3634007]
\end{abstract}

Optical binding presents an original method for selfarrangement of solid microparticles in liquid or air. ${ }^{1-4}$ The resulting equilibrium positions of the particles in an optically bound structure (OBS) are not only influenced by the spatial intensity profiles of the incident laser beams but also by the light scattered from all the bound particles. ${ }^{5}$ From a practical point of view, an OBS can be generated by simple experimental configurations that are commonly objective- or lensfree and well suited to integration with microfluidic systems. ${ }^{3,6-8}$ However, in contrast to widely spread multiple optical trapping based on computer generated holograms $\mathrm{s}^{9,10}$ or deflections of a trapping laser beam, such configurations do not allow individual positioning of each particle within the structure. ${ }^{11,12}$ The inter-particle distances in OBSs can be externally controlled by changing the refractive index of the medium $^{13}$ or the spatial intensity distribution of the counterpropagating $(\mathrm{CP})$ beams. ${ }^{14-16}$

In this paper, we present a method for dynamic size tuning of multi-dimensional OBSs via control of the spatial intensity profiles of the trapping beams. We dynamically modified the lateral intensity profiles of the incoherent CP beams using a flexible dual-beam setup based on a spatial light modulator (SLM). ${ }^{17}$ We show that an OBS can be stretched or squeezed along the direction of the optical axis within a wide range of the inter-particle distances, undergoing phase transitions if squeezed beyond a certain limit. We also show that a two-dimensional (2D) or three-dimensional (3D) OBS can be assembled and optically manipulated with a system of two CP Laguerre-Gaussian beams (LGBs) or several pairs of CP Gaussian beams (GBs), respectively. In both cases, the spatial arrangement of the OBS is conserved.

Our experimental system consisted of identical polystyrene (PS) microparticles dispersed in deionized water inside a glass capillary with an inner diameter of $100 \mu \mathrm{m}$ (Vitrocell 8510). The capillary was inserted into an optical field of CP laser beams and resulting OBSs were observed from a direction perpendicular to the axis of the laser beams. Individual beams (vacuum wavelength of $1064 \mathrm{~nm}$ ) were synthesized on an SLM (Hamamatsu LCOS X10468-07) in the form of

\footnotetext{
a)Electronic mail: zemanek@isibrno.cz.
}

zero-order Bessel beams (BBs), ${ }^{18} \mathrm{GBs}$, or LGBs,${ }^{19}$ each with the required width of the beam transversal profile. The diffraction losses of different phase masks were determined from the laser beam intensity incident on the SLM and the diffracted intensity measured in air at the sample plane.

The following examples reveal that the size of the laser beams has a significant influence on inter-particle distances within an OBS. Figure 1 presents a systematic theoretical and experimental comparison of the inter-particle distance as a function of the radius $\rho_{0}$ of a BB with suppressed longitudinal intensity variations. ${ }^{20,21}$ Therefore, the final OBS shape does not depend significantly on the axial position in the BB. Following theoretical predictions based on the coupleddipole method, ${ }^{22}$ we changed $\rho_{0}$ and analyzed the interparticle distances of structures containing two and three

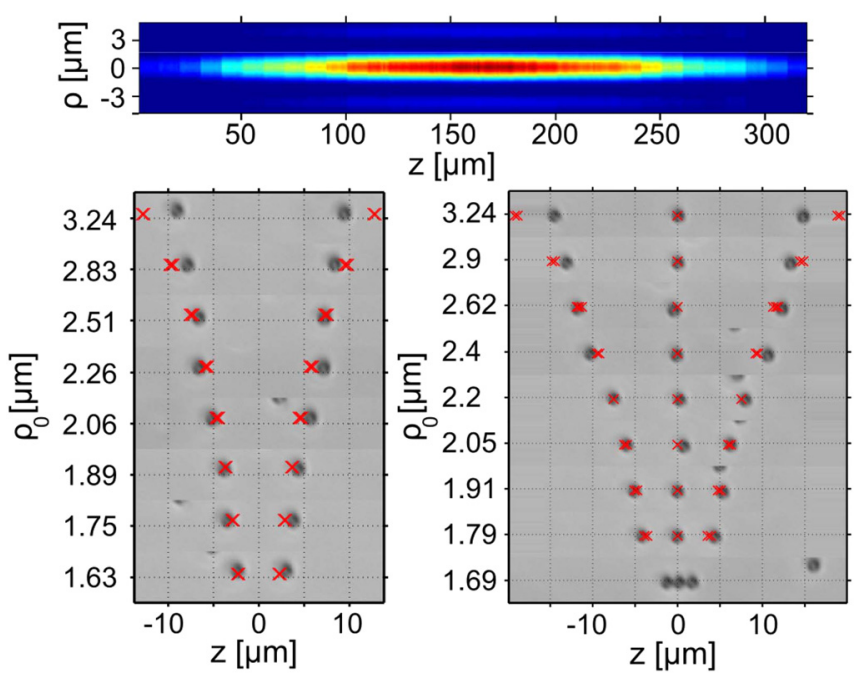

FIG. 1. (Color online) Optical binding in Bessel beams. Top: An example of the $\mathrm{BB}$ spatial intensity profile for the beam core radius $\rho_{0}=(1.67 \pm 0.03) \mu \mathrm{m}$. Bottom: Dynamic size tuning of an OBS made of polystyrene particles of diameter $1070 \mathrm{~nm}$ (Polysciences Inc.) is achived by varying the $\rho_{0}$ of two identical BBs counter-propagating along $z$ axis (the radii were determined to less than $5 \%$ error). The beam width decreases from top to bottom image rows. Laser power in the BB core incident on the OBS varied from $4 \mathrm{~mW}$ to $7 \mathrm{~mW}$ for the widest to narrowest beams, respectively. The grayscale images show the mean positions of the particles, red crosses denote the results of the theoretical predictions. The image on the bottom right (beam core radius $\rho_{0}=1.69 \mu \mathrm{m}$ ) illustrates a collapse of the OBS and indicates the OBS phase transition. 


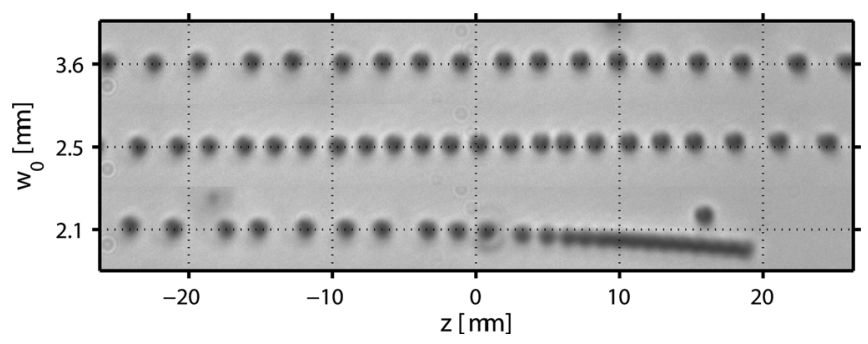

FIG. 2. Particle separation of an OBS comprising many particles is dynamically tuned by varying the waist radius $w_{0}$ of identical CP incoherent GBs. Each beam propagated along the $z$ axis ensuring lateral confinement of the PS particles (diameter $800 \mathrm{~nm}$ Duke Scientific). The incident powers of the wider and narrower beams varied between $20 \mathrm{~mW}$ and $40 \mathrm{~mW}$, respectively. The bottom row illustrates the collapse (phase transition) of the OBS in the narrowest beam and the consequent escape of the particles.

self-arranged particles. The agreement between the experimentally observed inter-particle distances and the theory is reasonably good for smaller inter-particle distances. The deviations for larger inter-particle distances may be caused by imperfections in the axial intensity profile of the BBs.

The $\mathrm{BB}$ represents an advantageous choice if one requires particles self-arrangement over long distances. However, in this situation, clustering of optically bound particles can occur. ${ }^{15}$ In contrast, wide CP GBs are easier to generate and can also provide non-clustered self-arranged chains of large numbers of particles. Figure 2 illustrates that modification of the GB waist radii also leads to squeezing or stretching of an OBS comprising many particles. Squeezing of OBSs occurs in narrower beams that push particles closer together untill the OBS becomes unstable, collapses, and a phase transition occurs. In contrast, we have not observed OBS collapse as the beams waist radii increase.

Experiments presented in Figs. 1 and 2 used radially symmetric laser beams that produced only one-dimensional OBS. However, we also obtained 2D OBSs existing in bulk liquid, far from any surfaces by using elliptical CP incoherent GBs. Up to now, OBSs in 2D have been observed only near a surface using CP evanescent waves ${ }^{23-25}$ or wide GBs. ${ }^{26}$ We kept the beam ellipticity constant (beam waist ratio $w_{x} / w_{y}=5$ ) which gave the theoretical estimate of the ratio of on-axis optical trap stiffness $\kappa_{x} / \kappa_{y}=1 / 25$. This allowed a larger separation of the particles along the $x$ axis and, subsequently, led to off-axis localization of the particles due to transverse optical binding.

Figure 3 presents the 2D self-arrangement of several particles far from any surface and illustrates direct proportionality between the beam waist size and the increase of inter-particle distance $(1.8 \times$ for the data shown $)$. In the case of OBS squeezing, this procedure enables further studies of phase transitions in colloidal structures.

Modification of phase mask at the SLM enables the generation of several pairs of $\mathrm{CP}$ incoherent GBs with variable beam waists. Figure 4 presents particle self-organization in three pairs of $\mathrm{CP}$ GBs with radially symmetric profiles whose axes were arranged laterally at the vertices of an equilateral triangle. Each image corresponds to a different beam waist radii while the distance between the axes of the beam pairs was constant. Similarly to Fig. 2, the inter-particle

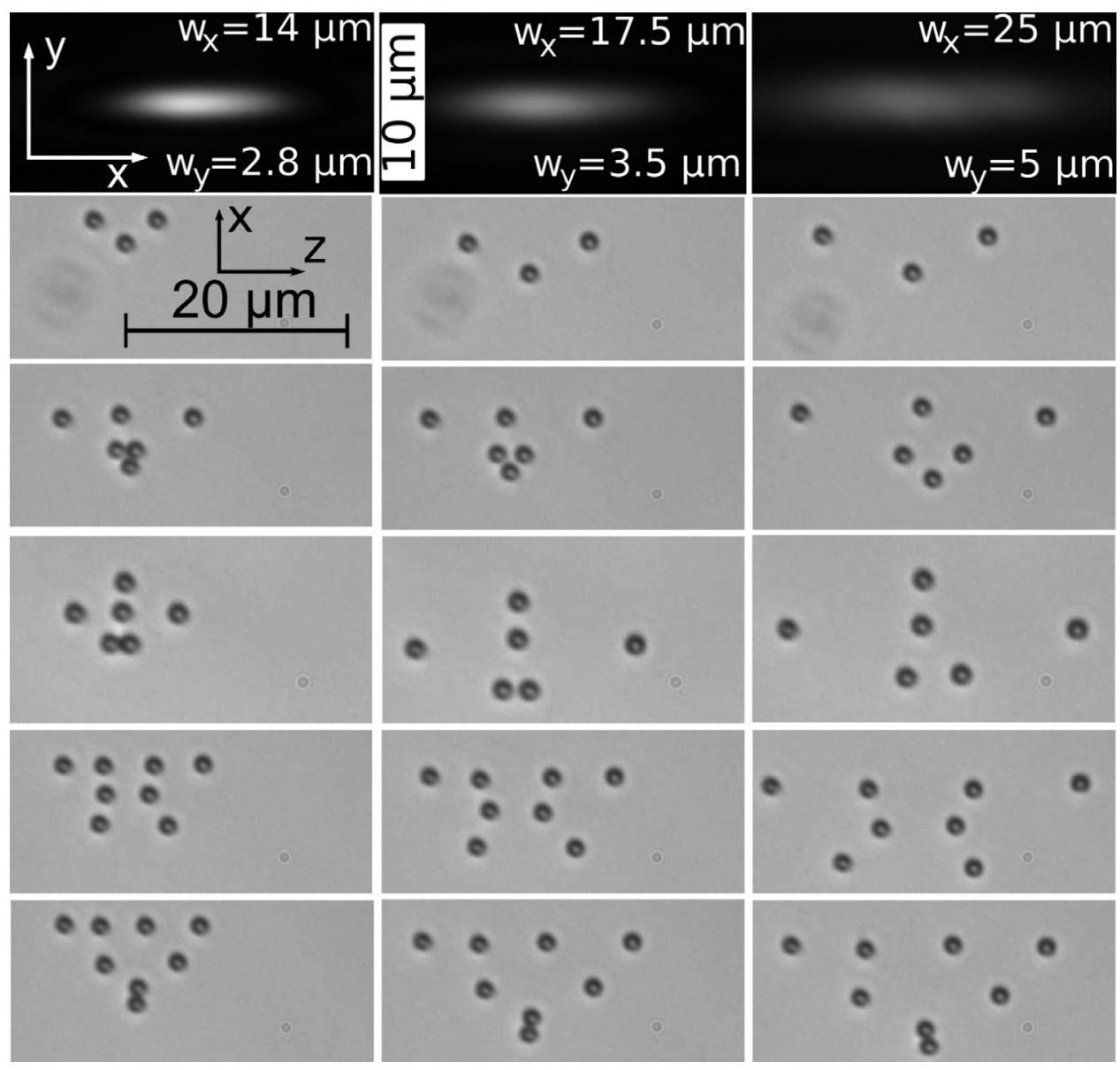

FIG. 3. Size control of 2D optically bound matter. The first row shows the beam waist profiles of three different elliptical Gaussian beams used in the study. The following rows show dynamic size tuning of 2D OBSs with various initial configurations of particles (1070 $\mathrm{nm}$ in diameter) self-arranged in the above depicted beams. The total power of a single beam at the sample plane varied between $80 \mathrm{~mW}$ and 35 $\mathrm{mW}$ for the narrowest and widest beam, respectively. 

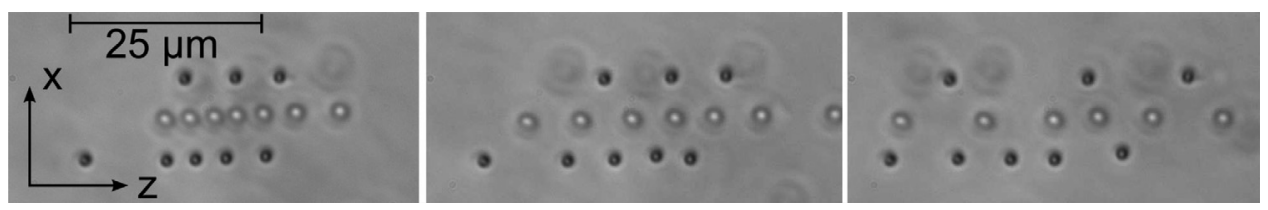

FIG. 4. Dynamic size tuning of 3D OBSs using three pairs of CP incoherent GBs. The diameter of PS particles was $1070 \mathrm{~nm}$, the waist radii of all GBs were subsequently set to $2.25 \mu \mathrm{m}, 2.75 \mu \mathrm{m}$, and $3.25 \mu \mathrm{m}$ (left to right). Fixed lateral separation between the beam pair axes was set by the SLM to $5.1 \mu \mathrm{m}$ and the total power in each GB was $20 \mathrm{~mW}$.

distances in the OBSs depended on the sizes of the beam waists and also on the number of particles arranged in each beam pair. Aside from the longitudinal binding, there was an optical interaction between particles confined in different pairs of CP GBs that led to correlated behavior of particles across the beam pairs. Therefore, several pairs of CP GBs indeed enable formation of tunable optically bound threedimensional structures.

The presented concept of OBS size tuning is applicable also to optical vortices and particles in motion. Figure 5 introduces an example of a 2D OBS revolving around the optical axis of CP incoherent LGBs of opposite topological charges \pm 1 . The angular momentum coming from one absorbed photon from each beam is equal to $2 \hbar$ and keeps the particles orbiting around the optical axis. ${ }^{27}$ However, we observed remarkably different behavior with a single particle, which showed no orbiting, compared to an OBS that revolved faster if more particles were confined in the structure. Besides optical binding, hydrodynamic effects should also be taken into account to fully describe this complex behavior. $^{28}$ Similarly to the static OBS case, comparison of OBSs revolving in beams of different sizes reveals that the size of the OBS is proportional to the beam waist radii and the OBS conserves its shape.

To date, only static OBSs have been investigated. Here, we have presented a method to change the dimensions of an OBS in real time by varying the lateral profile of the illuminating laser beams. We have also demonstrated phase transitions within the OBS for critical beam profile parameters. Such tool paves the way for series of new studies where the sizes of OBSs can be tuned to create self-arranged structures of appropriate size or to induce collapse (phase transition) of

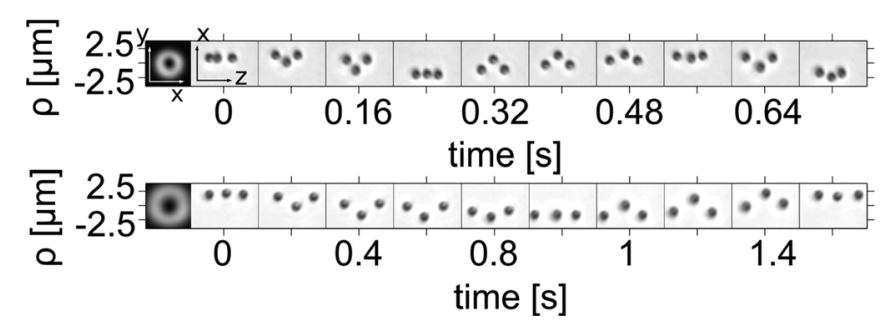

FIG. 5. Configurational dynamics of three PS particles of $1070 \mathrm{~nm}$ in diameter illuminated by $\mathrm{CP}$ incoherent LG beams of opposite topological charges \pm 1 . The beam waists were $2.2 \mu \mathrm{m}$ (top) and $3.4 \mu \mathrm{m}$ (bottom) with corresponding incident powers in a single beam $50 \mathrm{~mW}$ and $25 \mathrm{~mW}$, respectively. The duration of the single period of OBS revolution was $0.56 \mathrm{~s}$ and $1.8 \mathrm{~s}$ for the narrow and wide beams, respectively. the OBS into a closely packed colloidal crystal or a photonic band-gap structures even in 3D.

The authors acknowledge the support from CSF (202/ 09/0348; P205/11/P294) and valuable comments of A. Jonas and H. I. C. Dalgarno.

${ }^{1}$ M. M. Burns, J.-M. Fournier, and J. A. Golovchenko, Science 249, 749 (1990).

${ }^{2}$ S. A. Tatarkova, A. E. Carruthers, and K. Dholakia, Phys. Rev. Lett. 89, 283901 (2002).

${ }^{3}$ W. Singer, M. Frick, S. Bernet, and M. Ritsch-Marte, J. Opt. Soc. Am. B 20, 1568 (2003).

${ }^{4}$ M. Guillon, O. Moine, and B. Stout, Phys. Rev. Lett. 96, 143902 (2006).

${ }^{5}$ K. Dholakia and P. Zemánek, Rev. Mod. Phys. 82, 1767 (2010).

${ }^{6}$ S. K. Mohanty, K. S. Mohanty, and M. W. Berns, Opt. Lett. 33, 2155 (2008).

${ }^{7}$ D. M. Gherardi, A. E. Carruthers, T. Čižmár, E. M. Wright, and K. Dholakia, Appl. Phys. Lett. 93, 041110 (2008).

${ }^{8}$ J. Liu, Q.-F. Dai, X.-G. Huang, L.-J. Wu, Q. Guo, W. Hu, X.-B. Yang, S. Lan, A. V. Gopal, and V. A. Trofimov, Opt. Lett. 33, 2617 (2008).

${ }^{9}$ P. Rodrigo, V. Daria, and J. Glückstad, Appl. Phys. Lett. 86, 074103 (2005).

${ }^{10}$ M. Woerdemann, C. Alpmann, and C. Denz, Appl. Phys. Lett. 98, 111101 (2011).

${ }^{11}$ T. Čižmár, L. C. D. Romero, K. Dholakia, and D. L. Andrews, J. Phys. B 43, 102001 (2010).

${ }^{12}$ T. Čižmár, D. I. C. Dalgarno, P. C. Ashok, F. J. Gunn-Moore, and K. Dholakia, Appl. Phys. Lett. 98, 081114 (2011).

${ }^{13}$ N. K. Metzger, K. Dholakia, and E. M. Wright, Phys. Rev. Lett. 96, 068102 (2006).

${ }^{14}$ V. Karásek, K. Dholakia, and P. Zemánek, Appl. Phys. B 84, 149 (2006).

${ }^{15}$ V. Karásek, T. Čižmár, O. Brzobohatý, P. Zemánek, V. Garcés-Chávez, and K. Dholakia, Phys. Rev. Lett. 101, 143601 (2008).

${ }^{16}$ J. Rodriguez and D. L. Andrews, Opt. Lett. 33, 2464 (2008).

${ }^{17}$ T. Čižmár, O. Brzobohatý, K. Dholakia, and P. Zemánek, Laser Phys. Lett. 8, 50 (2011).

${ }^{18}$ T. Čižmár, M. Šiler, and P. Zemánek, Appl. Phys. B 84, 197 (2006).

${ }^{19}$ A. E. Siegman, Lasers (University Science Books, Sausalito, California, 1986).

${ }^{20}$ O. Brzobohatý, T. Čižmár, and P. Zemánek, Opt. Express 16, 12688 (2008).

${ }^{21}$ T. Čižmár and K. Dholakia, Opt. Express 17, 15558 (2009).

${ }^{22}$ V. Karásek, O. Brzobohatý, and P. Zemánek, J. Opt. A, Pure Appl. Opt. 11, 034009 (2009).

${ }^{23}$ C. D. Mellor, T. A. Fennerty, and C. D. Bain, Opt. Express 14, 10079 (2006).

${ }^{24}$ P. J. Reece, V. Garcés-Chávez, and K. Dholakia, Appl. Phys. Lett. 88, 221116 (2006).

${ }^{25}$ V. Garcés-Chávez, K. Dholakia, and G. C. Spalding, Appl. Phys. Lett. 86, 031106 (2005).

${ }^{26}$ O. Brzobohatý, T. Čižmár, V. Karásek, M. Šiler, K. Dholakia, and P. Zemánek, Opt. Express 18, 25389 (2010).

${ }^{27}$ N. B. Simpson, K. Dholakia, L. Allen, and M. J. Padgett, Opt. Lett. 22, 52 (1997).

${ }^{28}$ J. Ng, Z. Lin, and C. Chan, Phys. Rev. Lett. 104, 103601 (2010). 